\title{
Formal research of event ontology elements
}

\author{
Yajun Zhang ${ }^{1, a}$, Zongtian Liu ${ }^{1, b}$ and Yalan Zhang ${ }^{2, c}$
}

${ }^{1}$ School of Computer Engineering and Science, Shanghai University, Shanghai 200444, China

${ }^{2}$ Mechanical and electrical engineer division, Wenhua college, Hongshan district, Wuhan 430073, Hubei province, China

azyj1985email@163.com, bztliu@shu.edu.cn, cyalanz@126.com

Keywords: event ontology; event elements; formalization; Z language

\begin{abstract}
Events as the basic unit of human knowledge, reflect the movement of things, and compared with the static concept can be expressed more high-level semantic information, is more and more attention from researchers in recent years. Many researchers has done a lot of works to the definite event ontology system, but there are a lot of works needs to be for the related elements of formalization description. In this paper, using the $\mathrm{Z}$ language combined with a variety of formal representation, to formal and the elements of events ontology. From a series of formalization, this paper gives a set of suitable for event ontology elements formalized method. These will greatly enhance the event ontology description ability, at the same time also can make the existing event ontology with the basis of logical reasoning. It is a foundation to further the research on event ontology.
\end{abstract}

\section{Introduction}

Event is appreciable, relatively independent, moving existence. There is an essential difference between event and static concept[1]. The emergence and development of an event involves many aspects of the entity, these entity can be called event elements including action element, object element, time element, environment element,language performance. Starting from the event, we can define the set of common attribute event as event category or dynamic concept. Although reasonable definition of event is given, it is still hard for computer to handle texts and understand semantics by method of event. Main research purpose of this paper is studying formalization of every element of event ontology and formalization will lay a solid foundation for semantic analysis of texts based on events and event inference. There are many formal languages, and $\mathrm{Z}$ language, a formal language which is widely used, is used to study event related elements in this paper.

\section{Text Formal specification language and $\mathrm{Z}$ language}

The essence of formal method is one technology which describes the target software system properties based on mathematical technique. Formalized theory needs support of formal specification language. Formal specification language provides a system of notations named grammatical domain and precisely defined symbolic rules. There are many linguistic methods in the field of formal, such as description logic, VDM, Z language [2], B method and so on.

\section{Description Logic.}

Description logic, drawing main thoughts of KL-ONE, is formalization of knowledge representation based on object and a decidable subset of first-order logic. Except for knowledge representation, description logic is used in other domains[3], and it is the most important normalized form of object centered representation language. Main characteristic of description logic is very strong expressing ability and decidability and it can always ensure ceasing reasoning algorithm and return correct results. Among many well-known formalized methods of knowledge representation, special attention is paid to description logic in decades, main reasons for this are: they have clear mechanism of model theory; they are suitable for the concept taxonomy to represent the application domain; and they provide a lot of useful inference services[4]. 
But description logic are mainly used for processing hierarchical reasoning based on the concept, it is insufficient when processing dynamic knowledge based on events. Since concept is still and event is dynamic, the model of description logic can not describe and express the frame based on events.

\section{$\mathrm{Z}$ language.}

$\mathrm{Z}$ language is a kind of specification language using first order predicate calculus as main theoretical foundation and it is also one functional language[5]. The core of $Z$ language is $Z$-mode, which has two modes: state model and operation model. State mode defines a part of state space of goal software system and its constraint characteristic[6]. Operation mode describes the behavior characteristics of a part of the system; it can define one operating feature of the part by describing the relationship of state value of the part before and after operating. The model can be modified; the role of model modification is applying modifications to all variables in the declarations section.

Two modes of Z language can be expressed by figure1, Sname is mode's name, Decls is declaration part, Preds is predicate invariant part[7]. For state mode, declaration part is state variable's declaration, and predicate invariant is state variable's condition to be met. For operation mode, declaration part is state mode and input and output specification[8], and predicate invariant is operating condition and the change caused by the operation.

Predicate invariant of operation mode can be expressed as following.

Preds $=\operatorname{preOp}_{\wedge}$ postOp, preOp expresses state variable before operation and input condition to be met[9], that is pre-condition for operation; postOp expresses state variable after operation and input /output condition to be met[10], that is post -condition for operation.

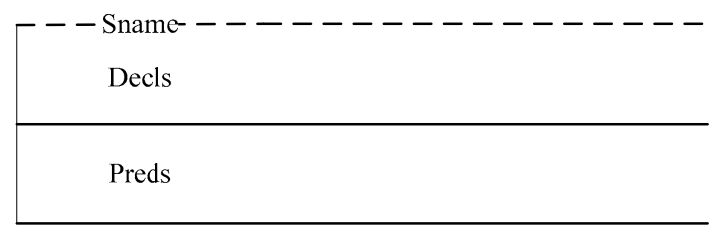

Figure1: Pattern framework of $Z$ language

\section{Formalization of event elements}

\section{Formalization of action element.}

Performing action mainly includes participants and concrete action. Participants can be divided into two parts, subject and object. Subject, who carries out action, is usually used as subject of sentence in the text.Object, who receives action, is usually used as object of sentence in the text. And action is described as predicate in the text, which is expressed by verb, connecting subject and object. Besides, act relationship between subject and object of sentence is contrary to relationship usually described for the reason of phrasing.

For instance, police arrests some illegal immigrant. This sentence is a typical subject-predicate-object structure sentence. Subject: police. Object: some illegal immigrant. Predicate: arrests. $Z$ language description of the action of this event is as shown in figure 2 . In this figure we define two participants: police,immigration. The former is subject, and the latter is object. Immigration status is defined as immigration's freedom, including two statuses: being free, being in harness. Immigration identity is defined as immigration's legal status, mainly including legal and illegal. Before police arresting them, immigration are illegal but they have freedom. After that, they are not free and still illegal. 


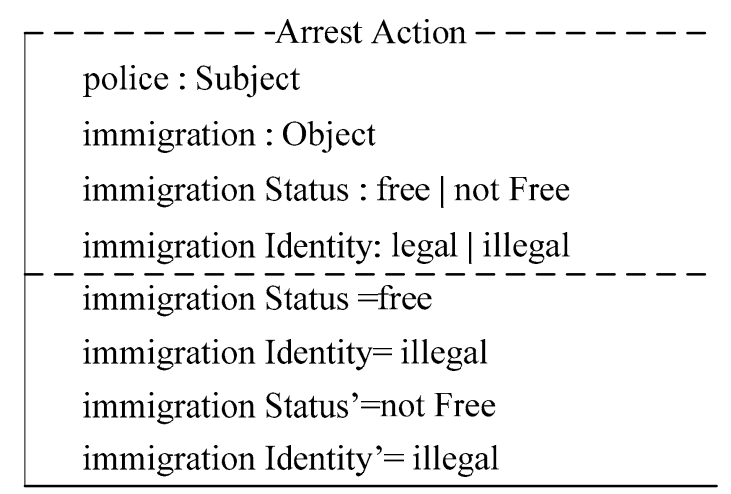

Figure2: Discreption of arrest action

\section{Formalization description of object elements.}

Object is main participation and leading role or the role to be changed of event. The target can be divided into subject and object. The subject is leading role, protagonist of event. In accordance with the division of labor, the object in the event divided into different roles, for example, in "earthquake" event, there are rescue personnel,rescue object,government sector,media. To special event class, object is constrained, for instance, rescue personnel in earthquake event can be doctors and army.

An example: Yilong Zheng, male, from Chaoyang district Shantou City Guangdong province. He is vice political director of vessel squadron of army police guangzhou detachment. In the afternoon of March 1 of 2013, he saved life of Chen yongbiao, a young man who fell into Zhujiang river, sadly Yilong Zhen was swept away by the rush of the current. In this event of policeman saving a young man'life, target subject factors can be formalized as figure 3, and target object factors can be formalized as figure 4.

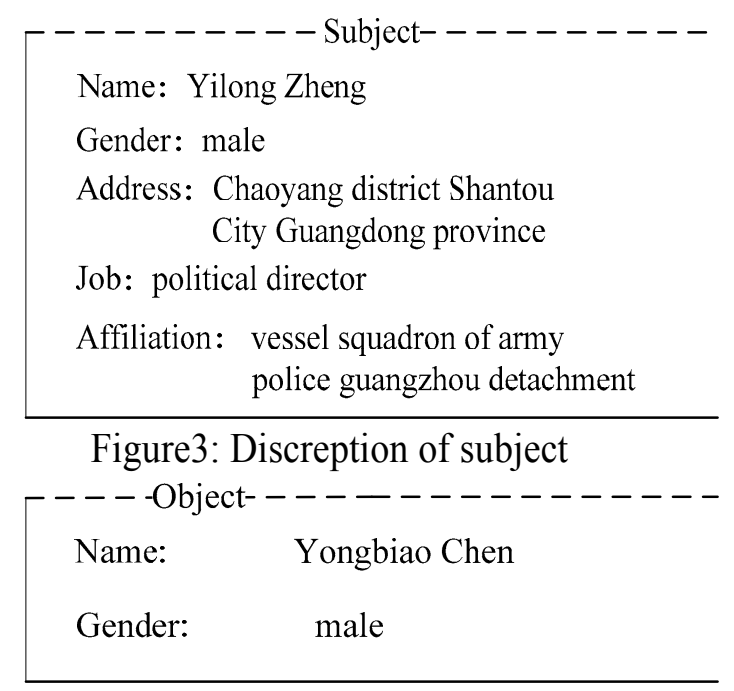

Figure4: Discreption of object

\section{Formalization description of time elements.}

Every event happens at certain time period, and time is one of most important factors of event evolving. Event developing usually go through beginning,developing,ending. Time period can be expressed by absolute time, and can be expressed by relative time, that means it can be expressed by relative bias.

Since event has two kinds of event: specific point of time event and time period event, so time can be divided into two kinds while describing the formalization of time. Time period can be expressed as type D, specific point of time can be expressed as type A.

For example: MH370 flight lost contact on March 8, 2014 at 1:20 in the morning. 


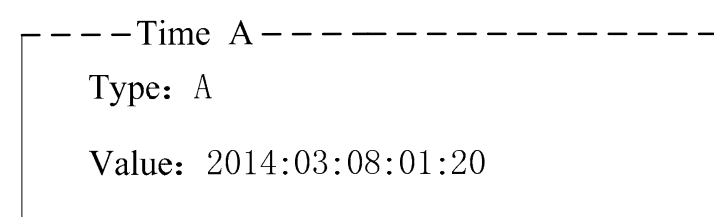

Figure 5: Description of specific point of time

For example: World War II broke out on September 1st of year 1939, starting with Germany invading Poland, on August 15th of year 1945 Japan announced unconditional surrender, which means World War II was officially over.

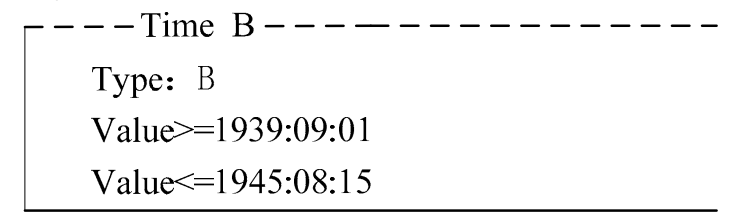

Figure 6: Description of time period

\section{Formalization of environment element.}

Any event must happen at certain environment. Environment consists of many entities according to certain relationships. Entities of environment factors are relatively still, which nearly doesn't change in the event, or even if the fact they change is not important.

In real world, contents of environment are rich, including concrete location, obscure region,real environment, virtual environment. We define abstract environment as type $\mathrm{V}$, and geography environment as type L.

An example : swimming in the water.

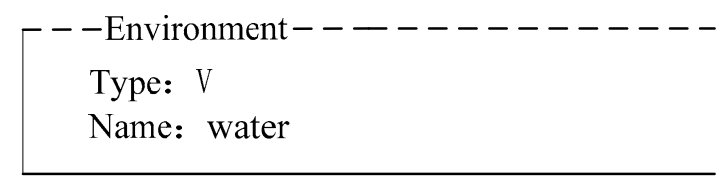

Figure 7: Description of abstract environment

Another example: In the morning there was a two-car collision in the direction of middle ring to Pudong airport, and five people were hurt, and 4 people was swept out of car, one person was at death's door.

$$
\begin{aligned}
& - \text { Environment }------------ \\
& \text { Type: } L \\
& \text { Name: middle ring to Pudong airport }
\end{aligned}
$$

Figure 8: Description of specific location environment

\section{Formalization description of language performance elements.}

To one event, different language has different performance; it also has different performance even if using same language. Language performance main content is core word. The core word is appellation of event and role of event, Language performance can be expressed by grammar, rule or template.

An example: the 5th international medicine exposition is hold in Taizhou city of Jiangsu province. Formalization description of core word is as shown in figure 9.

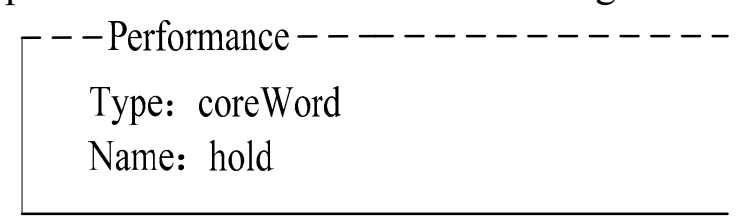

Figure 9: language performance description 


\section{Conclusion}

Firstly, concept of event ontology and advantage of it comparing to traditional ontology is introduced in this paper. And then there is brief introduction of formalization study. We analyze $\mathrm{Z}$ language used in this paper in detail. And we find that $Z$ language is very suitable for formalization description of event objects factors in event ontology. We give specific definitions of elements in event ontology including act,object,time,environment,language performance. This makes describing object elements in event ontology using $Z$ language comes true, laying a solid foundation for subsequent event ontology research. But the method in this paper has certain disadvantage, for instance, language performance formalization is hard to deal with, for the reason that definition of this element covers a wide range. And types of environment element should be subdivided. So we should do more research of these problems in future. What's more, we can make further research on formalization inference of event ontology after studying event element formalization is relatively sound.

\section{Acknowledgements}

We give sincere thanks to the persons, who jointly with us finished annotating event relation. This research was supported by the National Natural Science Foundation of China under Grant No.61273328, No.61305053 and No.71203135.

\section{References}

[1] Zongtian, L.; Meili, H.; Wen, Z.; Zhaoman, Z.; Jianfeng, F.; Jianfang, S. ; Huilai, Z. Research on Event-oriented Ontology Model Computer Science, 2009, 36, 189--192,199

[2] Duke R, Rose G, Smith G. Object-Z: a Specification Language Advocated for the Description of Standards[J]. Computer Standards \& Interfaces, 1995, 17(95):511-533.

[3] Smith G. The Object-Z Specification Language[J]. Advances in Formal Methods, 2000.

[4] Grosof B N, Horrocks I, Volz R, et al. Description Logic Programs: Combining Logic Programs with Description Logic[J]. General Information, 2003:48--57.

[5] Calvanese D, Lenzerini M, Nardi D, et al. Description logic framework for information integration[J]. Proc of the International Conference on Principles of Knowledge Representation \& Reasoning, 1998:2--13.

[6] Straccia U. A fuzzy description logic for the semantic web[C] Fuzzy Logic and the Semantic Web. Capturing Intelligence, Elsevier (2006) 73--90. 2005:594--599.

[7] Mike Spivey: The Z Notation: A Reference Manual, 2nd edition, Prentice Hall Inter-national Series in Computer Science, 1992.

[8] Jonathan Bowen: Formal Specification and Documentation using Z: A Case Study Approach, International Thomson Computer Press, International Thomson Publishing, 1996.

[9] Spivey. J. M. The Z notation: a reference manual, second edition 〔M International(UK) Ltd. 1992

[10] Steen M. W. A.. Consistency and composition of process specifications (D). PhD thesis, University of Kent at Canterbury, United Kingdom, 1998. 